\title{
Regra de Taylor e política monetária no Brasil: considerações empíricas a partir de um modelo DSGE para uma pequena economia aberta
}

\author{
Diego Ferreira*
}

\begin{abstract}
Resumo
O presente estudo tem como objetivo analisar a condução da política monetária brasileira após a adoção do sistema de metas de inflação. Para tal, avalia-se uma regra de Taylor estimada a partir de um modelo DSGE para uma pequena economia aberta. Comparam-se os resultados obtidos com os existentes na literatura nacional em busca de similaridades e/ou dissonâncias. As conclusões deste estudo são as seguintes: (i) a presença de rigidezes nominais e reais é significativamente relevante para a dinâmica da economia brasileira, com destaque à indexação de preços; (ii) o Banco Central do Brasil tem efetivamente uma postura anti-inflacionária; e (iii) a taxa de câmbio é um fator auxiliar na condução da política monetária nacional.
\end{abstract}

Palavras-chave: Política monetária. Regra de Taylor. Estimação bayesiana. DSGE.

Mestre pelo Programa de Pós-Graduação em Desenvolvimento Econômico (PPGDE) da Universidade Federal do Paraná (UFPR). E-mail: df.ferreiraa@gmail.com

http://dx.doi.org/10.5335/rtee.v21i44.5354

Submissão: 15/10/2014. Aceite: 05/03/2015. 


\section{Introdução}

Nos últimos anos, os modelos dinâmicos estocásticos de equilíbrio geral (DSGE) ${ }^{1}$ têm se consolidado como importantes ferramentas para avaliação de políticas econômicas sob o ambiente de economia aberta. Nesses modelos, equações comportamentais são explicitamente derivadas dos problemas de otimização intertemporal de agentes privados (famílias e firmas) sob restrições tecnológicas, orçamentárias e institucionais. Assim, as flutuações macroeconômicas seriam reflexos das respostas ótimas dos agentes aos choques de oferta e demanda em vários mercados.

Em contraste à abordagem keynesiana, cujas relações entre os agregados macroeconômicos são definidas de forma $a d$ hoc, a microfundamentação dos modelos DSGE faz com que eles não estejam tão propensos à crítica de Lucas $(1976)^{2}$, visto que tal framework captura os parâmetros estruturais atrelados a preferências e tecnologias. Além disso, conforme demonstraram Christiano, Eichenbaum e Evans (2005), a presença de imperfeições de mercado e de rigidez nominal seriam as responsáveis por reafirmar a não-neutralidade de políticas monetárias no curto-prazo.

De um ponto de vista pragmático, a satisfatória performance preditiva dessa classe de modelos ${ }^{3}$ fez com que eles se tornassem uma das principais ferramentas de avaliação econômica, não apenas na academia, mas também para os policy makers de todo o mundo ${ }^{4}$. Nesse sentido, resultados mais robustos acerca da dinâmica da economia seriam capazes de assegurar maior acurácia para o desenho de políticas monetárias e/ou fiscais.

No Brasil, o número de estudos desenvolvidos com base nos modelos DSGE ainda é escasso, apesar de crescente. Silveira (2006) baseia-se na construção de um modelo DSGE para dois países, calibrado com os parâmetros estruturais da economia brasileira, em que considera os termos de troca diretamente na curva de Phillips e a hipótese de viés doméstico nas preferências dos consumidores, criando assim um canal alternativo para a política monetária. Além disso, no trabalho de Valli e Carvalho (2010) foram analisados os impactos econômicos advindos da coordenação de políticas fiscais e monetárias para o Brasil, afirmando que cada instrumento fiscal apresenta impactos diferentes sobre a dinâmica do modelo.

O estudo de Castro et al. (2011), responsável pela construção efetiva do modelo Samba (Stochastic Analytical Model with a Bayesian Approach), desponta como o trabalho de maior relevância na literatura brasileira acerca dos modelos DSGE. Nesse, além da inclusão de características da economia nacional, buscou-se implementar regras tanto para a política fiscal quanto para a monetária, além de famílias não ricardianas. 
Dentre os estudos recentes, Araújo e Andrade (2012) estimaram um modelo DSGE com o intuito de analisar a curva de juros no Brasil. Por meio do estimador de momentos generalizados (GMM), os autores identificaram os parâmetros estruturais do modelo, enquanto o modelo de vetores autorregressivos (VAR) foi utilizado para obter as respostas contemporâneas da curva de juros aos choques macroeconômicos. Deve-se ressaltar também o estudo de Palma e Portugal (2012), que utilizaram um modelo DSGE para estimar as preferências do Banco Central brasileiro, sob um ambiente de discricionariedade e uma autoridade monetária que minimiza uma função perda quadrática, distintamente dos demais trabalhos que modelaram o comportamento do Banco Central por meio de uma regra de Taylor.

A existência de um pass-through cambial imperfeito sob os preços domésticos ${ }^{5}$, além de acentuada vunerabilidade externa brasileira, faz com que a taxa de câmbio apresente impactos substanciais sobre a determinação da taxa de juros (Ferreira, 2004). Logo, a inclusão do câmbio na função de reação do Banco Central do Brasil permitiria avaliar em mais detalhes as respostas da autoridade monetária nacional frente ao comportamento de tal variável.

Entretanto, a literatura DSGE brasileira ainda não tem amplamente se preocupado com os impactos do câmbio sobre a determinação da taxa básica de juros. Furlani, Portugal e Laurini (2008) podem ser considerados os únicos a avaliar efetivamente a influência cambial sobre a reação do Banco Central. Apesar de concluir-se que não há evidências estatisticamente significativas para respostas ao câmbio, investigações mais detalhadas são necessárias. Nesse sentido, o presente estudo incorpora não a taxa de câmbio per se, mas sua variação na função de reação do Banco Central com o intuito de avaliar as respostas da taxa básica de juros frente à volatilidade cambial.

Com base nos estudos de Galí e Monacelli (2005), Monacelli (2005) e Justiniano e Preston (2010), modelam-se a economia doméstica e a economia externa. Dessa forma, pretende-se avaliar a política monetária no Brasil, além de comparar os resultados das estimações obtidos com outros presentes na literatura nacional e internacional.

O restante deste estudo está organizado como segue: seção 2, apresenta-se o modelo DSGE a ser estimado; seção 3, discute-se a fonte dos dados, a calibração dos parâmetros e a escolha das distribuições a priori; seção 4, expõe os resultados obtidos para as estimações bem como suas discussões; seção 5 , é dedicada às conclusões.

\section{0 modelo DSGE para uma pequena economia aberta}

O modelo utilizado pelo presente trabalho parte dos estudos de Galí e Monacelli (2005), Monacelli (2005) e Justiniano e Preston (2010). Tal modelo foi desenvolvido para representar a dinâmica de uma pequena economia aberta, além 
de incorporar determinação alternada de preços à la Calvo (1983) e uma política monetária endógena (i.e. a taxa de juros é o instrumento monetário).

A estrutura do modelo pode ser sintetizada a partir de quatro agentes principais: (i) as famílias, (ii) as firmas produtoras domésticas, (iii) as firmas importadoras, (iv) a autoridade monetária doméstica e (v) a economia externa, composta por agentes análogos à economia doméstica. A Figura 1 representa a estrutura do modelo e as relações entre seus agentes ${ }^{6}$.

Figura 1 - Estrutura do modelo DSGE a ser estimado

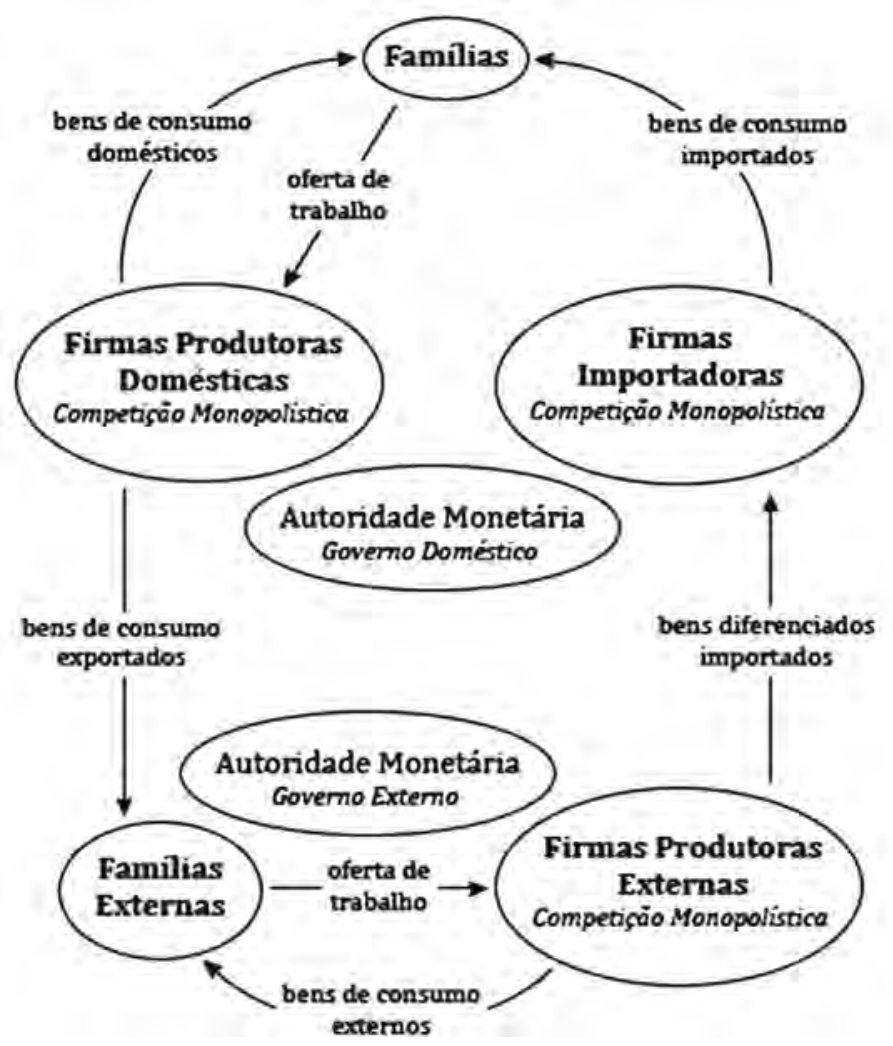

Fonte: elaboração do autor.

As famílias são responsáveis por ofertar trabalho às firmas produtoras domésticas, além de serem consumidoras de bens nacionais e importados, sendo suas decisões baseadas em um ambiente de maximização intertemporal de funções de utilidade, com expectativas do tipo forward-looking. Ressalta-se que a persistência de hábito no consumo é considerada a fonte de rigidez real no modelo. 
Tanto as firmas domésticas quanto as importadoras estabelecem seus preços em um ambiente de competição monopolística, com determinação alternada de preços, de acordo com Calvo (1983). As firmas domésticas são responsáveis por fornecer bens de consumo produzidos nacionalmente às famílias, bem como exportar seus bens de consumo às famílias externas, enquanto as firmas importadoras seriam apenas agentes responsáveis pela redistribuição de bens importados. Além disso, assume-se a existência de pass-through imperfeito da taxa de câmbio, considerada uma característica inerente à economia brasileira, bem como outra fonte de rigidez de preços.

A autoridade monetária doméstica é representada a partir de uma função de reação à la Taylor, em que se determina a taxa de juros como resposta à dinâmica da inflação, do produto, da variação da taxa real de câmbio e a um componente autorregressivo de primeira ordem.

Segundo Monacelli (2005), a economia externa é modelada como uma versão fechada da pequena economia. Todavia, diferente desse, foram considerados parâmetros específicos para a economia doméstica e a economia externa. Tem-se que os encadeamentos da economia externa sobre a economia doméstica ocorrem por meio da comercialização de bens diferenciados às firmas importadoras, bem como a demanda das famílias externas por produtos domésticos. Ressalta-se a existência de risk sharing imperfeito ${ }^{7}$.

No caso da autoridade monetária externa, a regra de Taylor estabelecida apenas responde à dinâmica da inflação do produto e a um componente autorregressivo de primeira ordem. Visto que o presente estudo baseia-se em Monacelli (2005), tal escolha para a função de reação externa justifica-se no fato de a economia externa ser modelada como uma versão fechada da economia doméstica.

\subsection{As famílias}

Seja um contínuo de famílias que vivem infinitamente, pertencentes ao intervalo $[0,1]$. $\mathrm{O}$ agente representativo enfrenta um problema de maximização da utilidade, visto que essa é dada por:

$$
U\left(C_{t}, H_{t}, N_{t}\right) \equiv \frac{\left(C_{t}-H_{t}\right)^{1-\sigma}}{1-\sigma}-\frac{N_{t}^{1+\varphi}}{1+\varphi}
$$

Em que $C_{t}$ é um índice de composição do consumo de bens; $H_{t}$ representa a formação de hábito externo, gerado por um processo autorregressivo de primeira ordem, com parâmetro $h ; N_{t}$ são as horas trabalhadas; $\sigma>0$ é o inverso da 
elasticidade de substituição intertemporal; e $\varphi>0$ é o inverso da elasticidade de oferta de trabalho.

O índice de consumo de bens $\left(C_{t}\right)$ é representado como uma combinação entre o consumo de bens domésticos e o consumo de bens importados por meio de uma função de elasticidade de substituição constante (CES):

$$
C_{t} \equiv\left[(1-\alpha)^{1 / \eta} C_{H, t}^{(\eta-1) / \eta}+\alpha^{1 / \eta} C_{F, t}^{(\eta-1)} / \eta\right]^{\eta /(\eta-1)}
$$

Em que $\alpha \in[0,1]$ é (inversamente) relacionado ao grau de viés doméstico nas preferências, tornando-se um índice natural para o grau de abertura da economia; $\eta>0$ mede a substitubilidade entre bens domésticos e importados, do ponto de vista do consumidor doméstico; e $C_{H, t}$ e $C_{F, t}$ são índices de consumo de bens domésticos e importados, respectivamente, representados também por funções CES:

$$
\begin{aligned}
C_{H, t} & \equiv\left[\int_{0}^{1} C_{H, t}(j)^{(\varepsilon-1)} / \varepsilon d j\right]^{\varepsilon /(\varepsilon-1)} \\
C_{F, t} & \equiv\left[\int_{0}^{1} C_{i, t}(j)^{(\gamma-1) / \gamma} d i\right]^{\gamma /(\gamma-1)}
\end{aligned}
$$

Sendo que $\varepsilon-1$ é a elasticidade de substituição entre as variedades de produtos; e $\gamma$ mede a substitubilidade entre bens produzidos em diferentes países.

Ressalta-se que $C_{i, t}$ corresponde a quantidade de bens importados do país $i$ e consumidos pela economia doméstica. Tal relação pode ser expressa também por:

$$
C_{i, t} \equiv\left[\int_{0}^{1} C_{i, t}(j)^{(\varepsilon-1) / \varepsilon} d j\right]^{\varepsilon /(\varepsilon-1)}
$$

O problema de otimização das famílias determina as seguintes funções ótimas de demanda:

$$
\begin{gathered}
C_{H, t} \equiv(1-\alpha)\left(\frac{P_{H, t}}{P_{t}}\right)^{-\eta} C_{t} \\
C_{F, t} \equiv \alpha\left(\frac{P_{F, t}}{P_{t}}\right)^{-\eta} C_{t}
\end{gathered}
$$

Em que $P_{H, t}$ e $P_{F, t}$ são os índices de preços domésticos e de bens importados, respectivamente, e expressos em moeda doméstica, dados por: 


$$
\begin{aligned}
& P_{H, t} \equiv\left[\int_{0}^{1} P_{H, t}(j)^{(1-\varepsilon)} d j\right]^{1 /(1-\varepsilon)} \\
& P_{F, t} \equiv\left[\int_{0}^{1} P_{i, t}(j)^{(1-\gamma)} d j\right]^{1 /(1-\gamma)}
\end{aligned}
$$

Logo, o índice de preço ao consumidor (IPC) é obtido ao substituir as funções de demanda no índice de consumo $\left(C_{t}\right)$ :

$$
P_{t} \equiv\left[(1-\alpha) P_{H, t}^{(1-\eta)}+\alpha P_{F, t}^{(1-\eta)}\right]^{1 /(1-\eta)}
$$

Portanto, a aproximação log-linear da equação de Euler para o consumo doméstico é dada por:

$$
c_{t}-h c_{t-1}=\left(\mathbb{E}_{t}\left\{c_{t+1}\right\}-h c_{t}\right)-\frac{(1-h)}{\sigma}\left(i_{t}-\mathbb{E}_{t}\left\{\pi_{t+1}\right\}\right)
$$

\subsection{As firmas produtoras domésticas}

Seja uma firma doméstica, produtora de bens, com função de produção expressa por $Y_{H, t}(i)=\epsilon_{a, t} N_{H, t}(i)$ em que $Y_{t}(i)$ é a produção da $i$-ésima firma $(i \in[0,1])$ e $N_{t}(i)$ é a quantidade de trabalho utilizado. Além disso, ressalta-se que $\epsilon_{a, t}$ é um choque tecnológico exógeno na economia doméstica, seguindo um processo AR(1).

A competição monopolística entre as firmas surge como forma de inserção de rigidez nominal de preços ao modelo, de forma que essas atuam na determinação de preços de forma alternada a cada período. Assim, aquelas que não determinam novamente seus preços $\left(\theta_{H}\right)$ apenas os indexam à inflação passada. Logo, as firmas otimizadoras resolvem seu problema de maximização, expresso por:

$$
\max _{P_{H, t}(i)} \mathbb{E}_{t} \sum_{s=0}^{\infty} Q_{t, T} \theta_{H}^{T-t} Y_{H, T}(i)\left[P_{H, t}(i)\left(\frac{P_{H, t+s-1}}{P_{H, t-1}}\right)^{\delta_{H}}-P_{H, T} M C_{H, T}\right]
$$

Sujeito à restrição de demanda da $i$-ésima firma:

$$
Y_{H, T}(i)=\left[\frac{P_{H, t}(i)}{P_{H, T}}\left(\frac{P_{H, T-1}}{P_{H, t-1}}\right)^{\delta_{H}}\right]^{-\varepsilon}\left(C_{H, T}+C_{H, T}^{*}\right)
$$


Em que $\varepsilon>1$ é a elasticidade de substituição entre as variedades de produtos; $\delta_{H} \in[0,1]$ é o grau de inércia da inflação; e $M C_{H, t}$ é o custo marginal real no período t, dado por:

$$
M C_{H, t}=\frac{W_{t}}{\left(\epsilon_{a, t} P_{H, t}\right)}
$$

Em que $W_{t}$ é o salário nominal pago às famílias. Ressalta-se que a função do custo marginal real é obtida a partir da hipótese de existência de mercado de fatores homogêneos.

De seu problema de otimização, a solução log-linearizada para as firmas confere a curva de Phillips novo-keynesiana para a inflação dos produtores domésticos:

$$
\pi_{H, t}-\delta_{H} \pi_{H, t-1}=\beta\left[\mathbb{E}_{t}\left\{\pi_{H, t+1}\right\}-\delta_{H} \pi_{H, t}\right]+\lambda_{H}\left(m c_{H, t}\right)
$$

Em que os termos de troca são dados por $s_{t}=p_{F, t}-p_{H, t}$; a inflação doméstica é dada por $\pi_{H, t}=p_{H, t}-p_{H, t-1}$; e $\lambda_{H}$ representa um grupo de parâmetros dado por $\lambda_{H} \equiv\left(1-\theta_{H}\right)\left(1-\beta \theta_{H}\right) / \theta_{H}$. O custo marginal real, em sua forma log-linearizada, pode ser expresso por:

$$
m c_{H, t}=\varphi y_{t}-(1+\varphi) \epsilon_{a, t}+\alpha s_{t}+\frac{\sigma}{1-h}\left(c_{t}-h c_{t-1}\right)
$$

\subsection{As firmas importadoras}

As firmas importadoras são responsáveis por redistribuir bens diferenciados às famílias. Ao buscarem tais bens na economia externa, deparam-se com a lei do preço único. Entretanto, o processo de determinação de preços domésticos é regido por competição monopolística. Dessa forma, há um hiato de preços entre o valor pago à economia externa (competitivo) e o valor cobrado das famílias (competição monopolística). Esse hiato, em termos log-lineares, pode ser representado por:

$$
\psi_{F, t}=e_{t}+p_{t}^{*}-p_{F, t}
$$

Em que $\psi_{F, t}$ é o hiato de preços no período $t ; e_{t}$ é a taxa nominal de câmbio no período $t$; $p_{t}^{*}$ é o preço competitivo dos bens importados na economia externa no período $t$; e $p_{F, t}$ é o preço dos bens importados cobrados às famílias no período $t$.

O modelo utilizado no presente estudo considera que as firmas importadoras agem de forma análoga às firmas produtoras domésticas. Logo, a curva de Phillips novo-keynesiana para a inflação de bens importados é expressa por: 


$$
\pi_{F, t}-\delta_{F} \pi_{F, t-1}=\beta\left[\mathbb{E}_{t}\left\{\pi_{F, t+1}\right\}-\delta_{F} \pi_{F, t}\right]+\lambda_{F}\left(\psi_{F, t}\right)
$$

De forma que $\lambda_{F}=\left(1-\theta_{F}\right)\left(1-\beta \theta_{F}\right) / \theta_{F}$ e $\psi_{F, t}=m c_{F, t}$.

\subsection{A autoridade monetária doméstica ${ }^{8}$}

Para o presente estudo, a autoridade monetária tem uma meta inflacionária explícita. Suas políticas são desenhadas com base em uma função de reação estocástica à $l a$ Taylor, que responde à taxa de juros passada $\left(i_{t-1}\right)$, à inflação geral presente $\left(\pi_{t}\right)$, ao produto presente $\left(y_{t}\right)$ e à variação na taxa real de câmbio $\left(\Delta q_{t}\right)$ :

$$
i_{t}=\rho_{i} i_{t-1}+\left(1-\rho_{i}\right)\left(\psi_{\pi} \pi_{t}+\psi_{y} y_{t}+\psi_{q} \Delta q_{t}\right)+\epsilon_{M, t}
$$

Ao considerar que a meta de inflação estabelecida pela autoridade monetária mantém-se constante no tempo, essa é normalizada em zero na equação (12), visto que as variáveis são expressas como desvios em relação a sua média.

\subsection{Termos de troca, taxa real de câmbio e condição de paridade dos juros}

Conforme exposto, os termos de troca podem ser expressos como o hiato existente entre o preço dos bens importados e o preço dos bens domésticos, sendo $s_{t}=p_{F, t}-p_{H, t}$. Assim, diferenciando tal identidade no tempo, tem-se:

$$
\Delta s_{t}=\pi_{F, t}-\pi_{H, t}
$$

A taxa real de câmbio, em termos log-lineares, é tida como:

$$
q_{t}=e_{t}+p_{t}^{*}-p_{t}
$$

Ao considerar a condição de paridade do poder de compra (PPC), a log-linearização do índice de preço ao consumidor, exposto pela equação (7), gera:

$$
p_{t}=(1-\alpha) p_{H, t}+\alpha p_{F, t}=p_{H, t}+\alpha s_{t}
$$

Dessa forma, a inflação geral doméstica pode ser obtida com os diferenciais no tempo da equação (15): 


$$
\pi_{t}=(1-\alpha) \pi_{H, t}+\alpha \pi_{F, t}=\pi_{H, t}+\alpha \Delta s_{t}
$$

Logo, a manipulação algébrica das equações (14) e (15) confere uma relação entre a taxa real de câmbio e os termos de troca, dada por:

$$
q_{t}=\psi_{F, t}+(1-\alpha) s_{t}
$$

A condição de paridade descoberta dos juros é compreendida como:

$$
\left(\mathbb{E}_{t}\left\{q_{t+1}\right\}-q_{t}\right)+\epsilon_{q, t}=\left(i_{t}-\mathbb{E}_{t}\left\{\pi_{t+1}\right\}\right)-\left(i_{t}^{*}-\mathbb{E}_{t}\left\{\pi_{t+1}^{*}\right\}\right)
$$

Em que $i_{t}$ e $i_{t}^{*}$ são as taxas de juros nominais domésticas e mundiais, respectivamente; e $\epsilon_{q, t}$ é um choque exógeno, que segue um processo AR(1). Os componentes com asteriscos sobrescritos referem-se a variáveis externas.

\subsection{A economia externa}

Conforme Monacelli (2005), a economia externa é modelada como uma versão fechada da pequena economia. Essa é responsável por ofertar bens diferenciados às firmas importadoras domésticas. A formação dos preços de tais bens segue um comportamento de competição monopolística. Todavia, visto que Monacelli (2005) impõe à economia externa os mesmos parâmetros estruturais da contrapartida doméstica, o presente estudo avança na modelagem ao considerar que a economia externa apresenta parâmetros estruturais próprios.

Ressalta-se que as firmas produtoras nacionais exportam bens de consumo domésticos à economia externa. De forma análoga à economia doméstica, a equação de Euler para o consumo externo, em termos log-lineares, é dada por:

$$
y_{t}^{*}-\mathrm{h}^{*} y_{t-1}^{*}=\mathbb{E}_{t}\left\{y_{t+1}^{*}\right\}-\mathrm{h}^{*} y_{t}^{*}-\frac{\left(1-h^{*}\right)}{\sigma^{*}}\left(i_{t}^{*}-\mathbb{E}_{t}\left\{\pi_{t+1}^{*}\right\}\right)+\left(\epsilon_{G, t}^{*}-\mathbb{E}_{t}\left\{\epsilon_{G, t+1}^{*}\right\}\right)
$$

Sendo $h^{*}$ a formação de hábito no consumo externo; $\sigma^{*}>0$ a elasticidade de substituição intertemporal externa; $i_{t}^{*}$ é a taxa de juros externa; $\pi_{t}^{*}$ é a inflação externa; e $y_{t}^{*}$ a renda externa. Considera-se que $\epsilon_{G, t}^{*}$ seja um choque exógeno, seguindo um processo $\mathrm{AR}(1)$.

Como as famílias externas, as firmas produtoras externas seguem processos análogos à economia doméstica. Logo, a determinação de preços externa é dada por: 


$$
\pi_{t}^{*}-\delta^{*} \pi_{t-1}^{*}=\frac{\left(1-\theta^{*}\right)\left(1-\beta^{*} \theta^{*}\right)}{\theta^{*}}\left(m c_{t}^{*}\right)+\beta^{*}\left(\mathbb{E}_{t}\left\{\pi_{t+1}^{*}\right\}-\delta^{*} \pi_{t}^{*}\right)
$$

Em que o custo marginal externo $\left(m c_{t}^{*}\right)$ é expresso por:

$$
m c_{t}^{*}=\varphi^{*} y_{t}^{*}-\left(1+\varphi^{*}\right) \epsilon_{a, t}^{*}+\alpha s_{t}+\frac{\sigma^{*}}{\left(1-h^{*}\right)}\left(y_{t}^{*}-\mathrm{h}^{*} y_{t-1}^{*}\right)
$$

Sendo $\epsilon_{a, t}^{*}$ um choque tecnológico exógeno na economia externa, seguindo um processo $\operatorname{AR}(1)$.

Além disso, a autoridade monetária externa segue uma regra de Taylor, que responde à dinâmica da inflação externa $\left(\pi_{t}^{*}\right)$, ao produto externo $\left(y_{t}^{*}\right)$ e à taxa de juros do período anterior $\left(i_{t-1}^{*}\right)$. Assim:

$$
i_{t}^{*}=\rho_{i}^{*} i_{t-1}^{*}+\left(1-\rho_{i}^{*}\right)\left(\psi_{\pi}^{*} \pi_{t}^{*}+\psi_{y}^{*} y_{t}^{*}\right)+\epsilon_{M, t}^{*}
$$

Como na contrapartida doméstica, a meta de inflação externa foi considerada constante, sendo normalizada à zero na equação (22), visto que as variáveis são expressas como desvios de sua média amostral.

\subsection{O equilíbrio geral}

Para que o fechamento do modelo seja realizado, todos os mercados devem estar em equilíbrio. No caso do mercado de bens, o equilíbrio de mercado requer que:

$$
y_{t}=(1-\alpha) c_{H, t}+\alpha c_{H, t}^{*}
$$

A contrapartida externa para a demanda por bens domésticos, em sua forma log-linear, é dada por:

$$
c_{H, t}^{*}=-\eta\left(p_{H, t}-p_{t}^{*}\right)+c_{t}^{*}=\eta\left(s_{t}+\psi_{F, t}\right)+c_{t}^{*}
$$

Assim, a aproximação log-linear do equilíbrio no mercado de bens, em termos do produto doméstico, é dado por:

$$
(1-\alpha) c_{t}=y_{t}-\alpha \eta(2-\alpha) s_{t}-\alpha \eta \psi_{F, t}-\alpha y_{t}^{*}
$$

Além disso, há um equilíbrio simétrico para os produtores domésticos, em que esses determinam o preço comum $P_{H, t}$, no período $t$. De forma análoga, todas as firmas importadoras determinam simetricamente o preço comum $P_{F, t}$, no período $t$. 
Por fim, assume-se que a riqueza inicial das famílias é idêntica. Dessa forma, as decisões de consumo e de portfolio são idênticas a cada período, pois esses agentes se deparam com a mesma restrição orçamentária.

\subsection{Método de solução e estimação bayesiana}

O modelo DSGE exposto no presente estudo é considerado um sistema de expectativas racionais linear na forma:

$$
\begin{aligned}
& 0=A x_{t}+B x_{t-1}+C y_{t}+D z_{t} \\
& 0=E_{t}\left[F x_{t+1}+G x_{t}+H x_{t-1}+J y_{t+1}+K y_{t}+L z_{t+1}+M z_{t}\right] \\
& z_{t+1}=N z_{t}+\epsilon_{t+1} ; \quad E_{t}\left[\epsilon_{t+1}\right]=0
\end{aligned}
$$

Em que $x_{t}$ e $y_{t}$ são os vetores de estado endógenos, enquanto $z_{t}$ é um vetor de processos estocásticos exógenos. Logo, o equilíbrio recursivo é dado por:

$$
\left\{\begin{array}{l}
x_{t}=P x_{t-1}+Q z_{t} \\
y_{t}=\mathrm{R} y_{t-1}+U z_{t}
\end{array}\right.
$$

De tal forma que o equilíbrio descrito pelas matrizes $P, Q, \mathrm{R}$ e $U$ se mostre estável.

A inexistência de longas séries de dados, bem como o número excessivo de variáveis endógenas presente nos modelos DSGE, fazem com que os métodos econométricos usuais (e.g. Método de Máxima Verossimilhança e de Momentos Generalizados) não apresentem resultados robustos e significativos às estimações, sendo assim não adequados ao problema.

Em razão disso, Canova (2007) afirma que a inferência bayesiana tem despontado como o instrumental mais adequado ao problema, visto que esta supera os entraves decorrentes da singularidade nas matrizes de covariância das variáveis endógenas, bem como aqueles advindos da incerteza acerca do modelo efetivamente representar o processo gerador dos dados.

Em razão disso, todas as informações sobre o parâmetro $\theta$ estão contidas na distribuição a posteriori. Logo, tem-se que o Princípio da Verossimilhança sempre se mantém.

Deve-se ter em mente que a inferência bayesiana não é responsável por gerar uma única estimativa de $\theta$, mas sim uma distribuição posterior a esse. Dessa forma, utiliza-se tal distribuição para inferir acerca do comportamento de $\theta$. Além 
disso, pode-se partir para uma abordagem frequentista e realizar testes de hipóteses e criação de intervalos de confiança.

Dessa forma, em termos de densidades, tem-se:

$$
p\left(\theta \mid Y^{T}\right)=\frac{p(\theta) L\left(Y^{T} \mid \theta\right)}{\int_{\Theta} p(\theta) L\left(Y^{T} \mid \theta\right) d \theta} \propto p(\theta) L\left(Y^{T} \mid \theta\right)
$$

Em que $p(\theta)$ é a densidade $a$ priori e $p\left(\theta \mid Y^{T}\right)$ é a densidade $a$ posteriori. Seja $L\left(Y^{T} \mid \theta\right)$ uma função de $\theta$ em relação a um $Y^{T}$ conhecido, qualquer função proporcional à ela é referida como uma função de verossimilhança. Ressalta-se que o denominar em (30) é conhecido como a função densidade marginal da amostra, responsável por normalizar a densidade da distribuição a posteriori. Assim, a determinação das distribuições a posteriori consiste basicamente na aplicação do teorema de Bayes.

A função de verossimilhança pode ser computada pela representação de estado-espaço do modelo, com a equação de medida sendo responsável por vincular os dados observados e o vetor de estado. O equilíbrio recursivo do modelo DSGE, dado na equação (29), apresenta a seguinte representação de estado-espaço simplificada:

$$
\left\{\begin{array}{c}
S_{t+1}=\Gamma_{1} S_{t}+\Gamma_{2} \xi_{t+1} \\
Y_{t}=\Lambda S_{t}+\vartheta_{t}
\end{array}\right.
$$

Sendo $S_{t}=\left\{x_{t}, y_{t}\right\}, \xi_{t}$ é o vetor de inovações de estado, $Y_{t}$ é um vetor $k \times 1$ de variáveis observáveis, e $\vartheta_{t}$ é tido como erros de medida. As matrizes $\Gamma_{1}$ e $\Gamma_{2}$ são funções dos parâmetros estruturais do modelo (ou zes $P, Q, \mathrm{R}$ e $U$ ) e $\Lambda$ define a relação entre as variáveis observadas e as variáveis de estado. Ao considerar que as inovações de estado e os erros de medida são distribuídos normalmente, com média zero e variância-covariância $\Xi$ e $\Upsilon$ respectivamente, tem-se a função de verossimilhança:

$$
\log L\left(\theta \mid \Gamma_{1}, \Gamma_{2}, \Lambda, \Xi, \Upsilon\right)=\sum_{t-1}^{T}\left[\frac{N}{2} \log 2 \pi+\frac{1}{2} \log \left|\Omega_{t \mid t-1}\right|+\frac{1}{2} \sum_{t-1}^{T} \vartheta_{t}^{\prime} \Omega_{t \mid t-1}^{-1} \vartheta_{t}\right]
$$

Em que $\Omega_{t \mid t-1}=\Lambda^{\prime} \sum_{t \mid t-1} \Lambda+\Upsilon$ e $\sum_{t \mid t-1}=\Gamma_{1} \sum_{t-1 \mid t-1} \Gamma_{1}^{\prime}+\Gamma_{2} \Xi \Gamma_{2}^{\prime}$. A partir de valores de estado iniciais, $S_{0} \sim N\left(\bar{S}_{0}, \Sigma_{0}\right)$, a equação (32) pode ser obtida por meio do filtro de Kalman.

Seja a sequência $\left\{\theta^{j}\right\}_{1}^{N} \sim p\left(\theta \mid Y^{T}\right)$, a Lei dos Grandes Números garante que:

$$
E_{\theta}\left[g(\theta) \mid Y^{T}\right]=\frac{1}{N} \sum_{j=1}^{N} g\left(\theta^{j}\right)
$$

Na qual $g($.$) é uma função de interesse.$ 
Portanto, tem-se que a sequência $\left\{\theta^{j}\right\}_{1}^{N}$ deve ser repetida até se obter uma distribuição estacionária de $\theta^{j}$. Dentre os estudos empíricos acerca da estimação de modelos DSGE, o algoritmo de Metropolis-Hastings ${ }^{9}$ tem sido utilizado com tal finalidade. Neste, constrói-se uma cadeia de Markov com espaço de estados no espaço paramétrico $\theta$, de fácil simulação e com distribuição de equilíbrio exatamente idêntica à distribuição a posteriori. A plataforma Dynare, conjuntamente ao software Matlab $^{\circledast}$, é capaz de realizar tais procedimentos.

\section{Fonte e tratamento de dados}

O modelo DSGE discutido na seção anterior será estimado ${ }^{10}$ por intermédio de métodos bayesianos. Para tal, são utilizados dados da economia brasileira compreendidos entre o primeiro trimestre de 2000 até o segundo trimestre de 2012, totalizando cinquenta observações. Logo, os dados baseiam-se no período posterior à adoção do regime de metas de inflação.

As séries coletadas foram: (i) inflação doméstica $\left(\pi_{t}\right)$, medida pelo Índice Nacional de Preços ao Comsumidor Amplo (IPCA); (ii) taxa real de câmbio doméstica, em R\$/US\$ $\left(q_{t}\right)$; (iii) taxa de juros nominal, Selic anualizada $\left(i_{t}\right)$; (iv) produto interno bruto (PIB) doméstico real, a preços de mercado $\left(y_{t}\right)$; (v) inflação dos EUA, medida pelo Índice de Preços ao Consumidor (IPC) $\left(\pi_{t}^{*}\right)$; (vi) taxa de juros nominal dos EUA, fundos federais estadunidenses anualizada $\left(i_{t}^{*}\right)$; e (vii) PIB dos EUA $\left(y_{t}^{*}\right)$. Todos os dados estão disponíveis nos websites do Instituto Brasileiro de Geografia e Estatística (IBGE) e do Instituto de Pesquisa Econômica Aplicada (Ipeadata).

Como tratamento, todas as séries foram submetidas a ajustes para sazonalidade bem como suas tendências foram removidas por meio da aplicação do filtro de Hodrick-Prescott (HP). ${ }^{11}$ Ressalta-se que as séries estão expressas como desvios de sua média amostral.

\subsection{Calibração e distribuição a priori dos parâmetros}

No processo de estimação bayesiana, a escolha das distribuições a priori dos parâmetros do modelo é elementar. A inferência bayesiana busca atualizar as suposições iniciais acerca do comportamento dos parâmetros, condicionada aos dados observados, por meio da aplicação do Teorema de Bayes. ${ }^{12}$

De modo geral, o paradigma bayesiano está centrado na interpretação da probabilidade como uma medida condicional de incerteza. Porém, em contraste à 
abordagem frequentista, tem-se que os dados em si são considerados quantidades fixas, enquanto os parâmetros são tratados como variáveis aleatórias. Logo, torna-se possível incorporar probabilidades aos parâmetros.

Como consenso na literatura, parâmetros cujos domínios encontram-se no intervalo [0,1] utilizam distribuição beta, enquanto a distribuição gama inversa é selecionada para aqueles que se situam entre $[0, \infty)$ e a distribuição gama para os demais casos. No total, são estimados trinta parâmetros ${ }^{13}$.

As Tabelas 1 a 3 apresentam as descrições dos parâmetros a serem estimados bem como as distribuições, médias e desvios-padrão estabelecidos. A Tabela 1 refere-se aos parâmetros das preferências das famílias, da determinação de preços pelas firmas domésticas e importadoras, da política monetária e da economia externa. Na Tabela 2, expõe-se os parâmetros de persistência dos três choques exógenos serialmente correlacionados. Por fim, a Tabela 3 apresenta os desvios-padrão dos choques exógenos.

A escolha das distribuições a priori seguiu o procedimento subjetivo, em que se tem informações prévias acerca do comportamento dos parâmetros (prioris informativas), seja por intuição ou com base em pesquisas anteriores. Tal abordagem é usual nos trabalhos que estimam modelos DSGE por meio de técnicas bayesiana (SMETS; WOUTERS, 2003; VALLI; CARVALHO, 2010; CASTRO et al., 2011).

Primeiramente, os parâmetros referentes ao fator de desconto intertemporal doméstico $(\beta)$ e o grau de abertura da economia $(\alpha)$ foram calibrados em 0,99 e 0,45 , respectivamente. Com relação à economia externa, o fator de desconto intertemporal externo $\left(\beta^{*}\right)$, a indexação de preços externos $\left(\delta^{*}\right)$ e a fração de firmas não-reotimizadoras externas $\left(\theta^{*}\right)$ foram calibrados de acordo com o estudo de Walque, Smets e Wouters (2005) para a economia estadunidense. Os valores estabelecidos foram $\beta^{*}=0,99, \delta^{*}=0,30$ e $\theta^{*}=0,70$.

O processo de estimação dos parâmetros levou em conta 500 mil replicações do algoritmo Metropolis-Hastings ( $\mathrm{MH}$ ), com cinco cadeias paralelas. Além disso, uma rotina de otimização baseada em cadeias de Monte-Carlo foi utilizada para obter a moda da distribuição a posteriori, responsável por inicializar o método $\mathrm{MH}$. 
Tabela 1 - Parâmetros comportamentais domésticos e externos

\begin{tabular}{|c|c|c|c|}
\hline Símbolo & Distribuição & Média I DP(1) & Descrição \\
\hline \multicolumn{4}{|c|}{ Preferências domésticas } \\
\hline$\beta$ & --- & $0,99^{(2)}$ & Fator de desconto intertemporal doméstico \\
\hline$\alpha$ & --- & $0,45^{(2)}$ & $\begin{array}{l}\text { Grau de abertura (proporção de importações no consumo } \\
\text { doméstico) }\end{array}$ \\
\hline $\mathrm{h}$ & Beta & $0,70 \mid 0,10$ & Parâmetro de formação de hábito no consumo doméstico \\
\hline$\sigma$ & Gama & $1,00 \mid 0,20$ & Elasticidade inversa de substituição intertemporal doméstica \\
\hline$\varphi$ & Gama & $2,00 \mid 0,35$ & Elasticidade inversa de oferta de trabalho doméstica \\
\hline$\eta$ & Gama & $0,60 \mid 0,10$ & $\begin{array}{l}\text { Elasticidade de substituição entre bens domésticos e impor- } \\
\text { tados doméstica }\end{array}$ \\
\hline \multicolumn{4}{|c|}{ Determinação de preços domésticos } \\
\hline$\delta_{H}$ & Beta & $0,70 \mid 0,20$ & Backward-looking nos preços de bens domésticos \\
\hline$\delta_{F}$ & Beta & $0,70 \mid 0,20$ & Backward-looking nos preços de bens importados \\
\hline$\theta_{H}$ & Beta & $0,50 \mid 0,20$ & Fração de firmas domésticas não otimizadoras \\
\hline$\theta_{F}$ & Beta & $0,50 \mid 0,20$ & Fração de firmas importadoras não otimizadoras \\
\hline \multicolumn{4}{|c|}{ Política monetária doméstica } \\
\hline$\rho_{i}$ & Beta & $0,50 \mid 0,15$ & Parâmetro de suavização da taxa de juros doméstica \\
\hline$\psi_{\pi}$ & Gama & $1,50 \mid 0,30$ & Coeficiente da inflação na regra de Taylor doméstica \\
\hline$\psi_{y}$ & Gama & $0,25 \mid 0,10$ & Coeficiente do produto na regra de Taylor doméstica \\
\hline$\psi_{q}$ & Gama & $0,25 \mid 0,10$ & Coeficiente do câmbio na regra de Taylor doméstica \\
\hline \multicolumn{4}{|c|}{ Preferências externas } \\
\hline$\beta^{*}$ & --- & $0,99^{(2)}$ & Fator de desconto intertemporal externo \\
\hline$h^{*}$ & Beta & $0,70 \mid 0,10$ & Parâmetro de formação de hábito no consumo externo \\
\hline$\sigma^{*}$ & Gama & $1,00 \mid 0,20$ & Elasticidade inversa de substituição intertemporal externo \\
\hline$\varphi^{*}$ & Gama & $2,00 \mid 0,35$ & Elasticidade inversa de oferta de trabalho externo \\
\hline \multicolumn{4}{|c|}{ Determinação de preços externos } \\
\hline$\delta^{*}$ & --- & $0,30^{(2)}$ & Backward-looking nos preços de bens externos \\
\hline$\theta^{*}$ & --- & $0,70^{(2)}$ & Fração de firmas externas não otimizadoras \\
\hline \multicolumn{4}{|c|}{ Política monetária externa } \\
\hline$\rho_{i}^{*}$ & Beta & $0,50 \mid 0,15$ & Parâmetro de suavização da taxa de juros externa \\
\hline$\psi_{\pi}^{*}$ & Gama & $1,50 \mid 0,25$ & Coeficiente da inflação na regra de Taylor externa \\
\hline$\psi_{y}^{*}$ & Gama & $0,25 \mid 0,10$ & Coeficiente do produto na regra de Taylor externa \\
\hline
\end{tabular}

Fonte: Walque et al. (2005); Silva e Portugal (2010); e Palma e Portugal (2012).

(1) DP refere-se a desvio-padrão (2) Parâmetros calibrados 
Tabela 2 - Parâmetros de persistência dos choques domésticos e externos

\begin{tabular}{l|l|r|l}
\hline \multicolumn{1}{c|}{ Símbolo } & Distribuição & Média | DP(1) & \multicolumn{1}{c}{ Descrição } \\
\hline$\rho_{a}$ & Beta & $0,50 \mid 0,20$ & Persistência do choque de tecnologia doméstica \\
$\rho_{G}$ & Beta & $0,50 \mid 0,20$ & Persistência do choque de preferências domésticas \\
$\rho_{q}$ & Beta & $0,50 \mid 0,20$ & Persistência do choque da taxa de câmbio \\
$\rho_{a}^{*}$ & Beta & $0,50 \mid 0,20$ & Persistência do choque de tecnologia externa \\
$\rho_{G}^{*}$ & Beta & $0,50 \mid 0,20$ & Persistência do choque de preferências externas \\
\hline
\end{tabular}

Fonte: Walque et al. (2005); Silva e Portugal (2010); e Palma e Portugal (2012).

(1) DP refere-se a desvio-padrão

Tabela 3 - Desvios-padrão dos choques domésticos e externos

\begin{tabular}{l|l|r|l}
\hline \multicolumn{1}{c|}{ Símbolo } & Distribuição & Média | DP(1) & \multicolumn{1}{|c}{ Descrição } \\
\hline$\sigma_{a}$ & Gama inversa & $3,00 \mid \infty$ & Desvio-padrão do choque de tecnologia doméstica \\
$\sigma_{q}$ & Gama inversa & $1,00 \mid \infty$ & Desvio-padrão do choque de taxa de câmbio \\
$\sigma_{G}$ & Gama inversa & $1,00 \mid \infty$ & Desvio-padrão do choque de preferências domésticas \\
$\sigma_{M}$ & Gama inversa & $1,00 \mid \infty$ & Desvio-padrão do choque de taxa de juros doméstica \\
$\sigma_{a}^{*}$ & Gama inversa & $3,00 \mid \infty$ & Desvio-padrão do choque de tecnologia externa \\
$\sigma_{G}^{*}$ & Gama inversa & $1,00 \mid \infty$ & Desvio-padrão do choque de preferências externas \\
$\sigma_{M}^{*}$ & Gama inversa & $1,00 \mid \infty$ & Desvio-padrão do choque de taxa de juros externa \\
\hline
\end{tabular}

Fonte: Walque et al. (2005); Silva e Portugal (2010); e Palma e Portugal (2012).

(1) DP refere-se a desvio-padrão

\section{Resultados e discussões}

Por intermédio dos parâmetros comportamentais de um modelo DSGE, pode-se compreender em detalhes mais abrangentes a dinâmica dos agentes econômicos presentes em uma economia. Dessa forma, analisam-se os parâmetros domésticos de preferências das famílias e de determinação de preços das firmas com o objetivo de tecer comentários acerca das características da economia brasileira. Para isso, comparam-se as estimativas domésticas obtidas com os resultados de estudos DSGE selecionados, tanto nacionais quanto internacionais. Os resultados completos podem ser consultados na Tabela A, no Apêndice. 
Conforme apresentado na Tabela 4, o valor estimado para a elasticidade inversa de substituição intertemporal $(\sigma)$ permite inferir que as famílias brasileiras são altamente propensas a suavizar seu consumo. Com exceção de Silveira (2008), tal inferência é corroborada pelos demais estudos nacionais. Os estudos internacionais mostram que o Brasil possui um padrão de consumo intertemporal próximo às economias avançadas. Todavia, Castro et al. (2011) afirmam que a identificação desse parâmetro é geralmente difícil, podendo justificar a discrepância dos resultados.

Tabela 4 - Parâmetros comportamentais: estimativas e estudos DSGE selecionados

\begin{tabular}{|c|c|c|c|c|c|c|c|}
\hline \multirow{2}{*}{ Parâmetro } & \multirow{2}{*}{$\begin{array}{c}\text { Resultados } \\
\text { obtidos }\end{array}$} & \multicolumn{4}{|c|}{ Evidências nacionais } & \multicolumn{2}{|c|}{ Evidências internacionais } \\
\hline & & $\begin{array}{c}\text { Silveira } \\
(2008)\end{array}$ & $\begin{array}{c}\text { Castro et al. } \\
(2011)-\text { Samba }\end{array}$ & $\begin{array}{c}\text { Silva e } \\
\text { Portugal (2010) }\end{array}$ & $\begin{array}{c}\text { Palma e } \\
\text { Portugal (2012) }\end{array}$ & $\begin{array}{c}\text { Smets e Wouters } \\
\text { (2005) - UE }\end{array}$ & $\begin{array}{l}\text { Walque et al. } \\
(2005)-\text { EUA }\end{array}$ \\
\hline \multicolumn{8}{|c|}{ Preferências domésticas } \\
\hline$h$ & 0,7571 & 0,81 & 0,74 & 0,9562 & 0,89 & 0,61 & 0,72 \\
\hline$\sigma$ & 1,2646 & 2,86 & 1,30 & 1,2234 & 1,09 & 1,13 & 0,94 \\
\hline$\varphi$ & 1,7959 & 1,91 & $1,00^{(2)}$ & 1,8128 & 1,71 & 2,00 & 2,72 \\
\hline$\eta$ & 0,5221 & 0,11 & 0,10 & $--(3)$ & 0,13 & $---(5)$ & 0,57 \\
\hline \multicolumn{8}{|c|}{ Determinação de preços domésticos } \\
\hline$\delta_{H}$ & 0,3615 & & 0,57 & & 0,31 & & \\
\hline$\delta_{F}$ & 0,7967 & $0,71^{(1)}$ & 0,65 & $0,4005^{(3)}$ & 0,07 & $0,29^{(5)}$ & $0,28^{(6)}$ \\
\hline$\theta_{H}$ & 0,9904 & & 0,74 & & 0,66 & & \\
\hline$\theta_{F}$ & 0,8720 & $0,97^{(1)}$ & 0,64 & $0,4080^{(3)(4)}$ & 0,87 & $0,90^{(5)}$ & $0,70^{(6)}$ \\
\hline
\end{tabular}

Fonte: elaboração do autor.

(1) Presença de apenas um tipo de firma doméstica (2) A elasticidade inversa de oferta de trabalho ( $\varphi$ ) Foi calibrada (3) Estudo para economia fechada, portanto ausência de firmas importadoras ${ }^{(4)}$ A proporção de firmas não-reotimizadoras $(\theta)$ Foi calibrada (5) Estudo para economia fechada, portanto ausência de firmas importadoras ${ }^{\left({ }^{(6)}\right.}$ Presença de apenas um tipo de firma doméstica

Com relação às preferências domésticas das famílias, a formação de hábito no consumo $(h)$ mostra-se relevante para a dinâmica da economia brasileira, estimada em 0,7571. Tal resultado aproxima-se aos obtidos no modelo Samba (CASTRO et al., 2011) e no estudo realizado por Walque et al. (2005) para os EUA, estimados em 0,74 e 0,72, respectivamente. Em contraste aos demais, a União Europeia (SMETS; WOUTERS, 2005) apresentou menor grau de formação de hábito no consumo, com valor estimado $h=0,61$. Ressalta-se que o resultado obtido insere-se nos patamares presentes na literatura recente.

A estimativa para a elasticidade de substituição entre bens domésticos e importados $(\eta=0,5221)$ revela que as famílias brasileiras apresentam um padrão 
de baixa substitubilidade entre os bens em questão. Apesar dos demais estudos nacionais apontarem para valores inferiores, esse padrão ainda é corroborado. No entanto, o estudo de Walque et al. (2005) aponta evidências de que os EUA apresentam maior grau de substitubilidade entre bens domésticos e importados.

No que tange ao mercado de trabalho, o modelo aponta para um caráter de acentuada rigidez, visto que o valor estimado para a elasticidade de oferta de trabalho é baixo $(0,5568, \varphi=1,7959)$. Apesar de o Brasil ainda apresentar maior rigidez salarial do que os EUA $(0,37, \varphi=2,72)$, aproxima-se do padrão encontrado na União Europeia $(0,50, \varphi=2,00)$. Os demais estudos nacionais corroboram o resultado obtido.

De modo geral, o modelo demonstra a presença de acentuada indexação de preços (e consequente rigidez) na economia brasileira, sendo tal resultado corroborado pelo modelo Samba e pelo estudo de Silveira (2008). Mais especificamente, quando tratada tanto para as firmas produtoras domésticas quanto para as firmas importadoras, as estimativas revelam evidências de maior indexação nas firmas importadoras, como no modelo Samba.

Frente aos problemas macroeconômicos provindos de falhas na condução da reforma fiscal da crise mexicana em 1995, da crise asiática de 1997, e da moratória russa de 1998, o Banco Central brasileiro assume a responsabilidade de estabilização do nível de preços ao final dos anos 1990. A adoção do sistema de metas de inflação (inflation targeting) foi responsável por intensificar o debate acerca dos mecanismos de transmissão da política monetária doméstica, além da forma como a autoridade monetária responderia às flutuações dos agregados macroeconômicos.

Com o objetivo de contribuir com o entendimento acerca das respostas da taxa de juros básica brasileira (Selic) aos agregados macroecômicos, analisam-se os parâmetros estimados de uma regra de Taylor para o Brasil. A Tabela 5 apresenta os resultados obtidos, bem como as estimativas de estudos nacionais selecionados.

Tabela 5 - Parâmetros da política monetária brasileira: estimativas e estudos DSGE selecionados

\begin{tabular}{l|r|r|r|c|c|c|c}
\hline Parâmetro & $\begin{array}{c}\text { Resultados } \\
\text { obtidos }\end{array}$ & $\begin{array}{c}\text { Sin e Gaglianone } \\
(2006)\end{array}$ & $\begin{array}{c}\text { Silveira } \\
(2008)\end{array}$ & $\begin{array}{c}\text { Furlani, Portugal } \\
\text { e Laurini (2008)(1) }\end{array}$ & $\begin{array}{c}\text { Castro et al. } \\
(2011)-\text { Samba }\end{array}$ & $\begin{array}{c}\text { Silva e Portugal } \\
(2011)\end{array}$ & $\begin{array}{c}\text { Kanczuk } \\
(2012)\end{array}$ \\
\hline$\rho_{i}$ & 0,4363 & 0,8402 & 0,7400 & 0,8704 & 0,7900 & 0,8721 & 0,6100 \\
$\psi_{\pi}$ & 1,2540 & 1,3328 & 1,4500 & 4,1842 & 2,4300 & 1,6578 & 3,0500 \\
$\psi_{y}$ & 0,6441 & 0,1350 & 1,1500 & 5,8727 & 0,1600 & 0,1911 & 0,2200 \\
$\psi_{q}$ & 0,5455 & --- & --- & 4,5722 & --- & --- & --- \\
\hline
\end{tabular}

Fonte: elaboração do autor.

(1) Os resultados obtidos por Furlani, Portugal e Laurini (2008) afastam-se dos encontrados nos modelos DSGE nacionais devido à imposição de distribuições a priori com médias relativamente elevadas $(\mu=5)$ em comparação aos demais. 
As estimativas realizadas permitem concluir que o Banco Central brasileiro segue uma política anti-inflacionária, visto que $\psi_{\pi}=1,2540$. Dessa forma, a atuação da autoridade monetária brasileira estaria de acordo com as diretrizes do sistema de metas de inflação. Com exceção de Furlani, Portugal e Laurini (2008), as evidências empíricas dos demais estudos corroboram o caráter central anti-inflacionário das políticas monetárias brasileiras. Já o valor estimado para o parâmetro de reação ao produto $\left(\psi_{y}=0,6441\right)$ reflete uma possível recente preocupação da autoridade monetária brasileira com a manutenção do crescimento econômico.

Entretanto, o coeficiente de suavização da taxa de juros $\left(\rho_{i}\right)$, estimado em 0,4363, demonstra um padrão de persistência (suavidade) menos expressivo do que o encontrado nos demais estudos nacionais. Em geral, os bancos centrais tendem a suavizar a fixação da taxa básica de juros na busca por maior credibilidade na política monetária, bem como para manter uma inércia nos juros a fim de afetar as taxas de horizonte mais longo (GOODFRIEND, 1991; CLARIDA, GALÍ, GERTLER, 1999).

Por fim, os resultados do modelo permitiram visualizar que o Banco Central aparentemente responde a variações na taxa real de câmbio $\left(\psi_{q}=0,5455\right)$. Ainda assim, a resposta da autoridade monetária brasileira ao câmbio é menos expressiva do que a preocupação com o crescimento econômico e o controle inflacionário, visto que $\psi_{\pi}>\psi_{y}>\psi_{q}$. De acordo com Ferreira (2004), a vulnerabilidade externa brasileira, atrelada à existência de um significativo pass-through cambial, é a responsável por fazer com que a taxa de câmbio apresente expressiva influência sobre a taxa básica de juros.

\section{Considerações finais}

Ao final da década de 1990, com a adoção do sistema de metas de inflação, tornou-se imperativo analisar a condução da política monetária brasileira. Por intermédio de uma regra de Taylor, aproxima-se o comportamento do Banco Central diante de flutuações dos agregados macroeconômicos. O presente estudo insere-se na discussão acerca das estimativas para os parâmetros da função de reação da autoridade monetária brasileira, ao comparar os resultados obtidos com outros estudos DSGE nacionais.

Com base nos modelos de Monacelli (2005), Galí e Monacelli (2005) e Justiniano e Preston (2010), os parâmetros estruturais brasileiros são estimados com base em técnicas bayesianas consideradas padrão na literatura DSGE. A partir desses, pôde-se tecer comentários referentes à dinâmica da economia brasileira. 
Em relação à aderência dos parâmetros comportamentais às demais estimativas presentes na literatura nacional e internacional, o modelo apresentou resultados consistentes. Pôde-se concluir que as fontes de rigidezes nominais e reais incluídas foram significativamente relevantes para a dinâmica do modelo, com destaque para a indexação de preços.

A condução da política monetária, pela regra de Taylor, também foi avaliada. Concluiu-se que o Banco Central brasileiro possui uma postura anti-inflacionária, em acordo com os preceitos do sistema de metas de inflação. Tal resultado é corroborado pelos demais estudos nacionais. Ainda, a inclusão da taxa de câmbio permitiu visualizar que a volatilidade cambial é utilizada como fator auxiliar na determinação da taxa básica de juros, apesar dos componentes referentes à inflação e ao produto serem mais expressivos. Entretanto, ressaltou-se a necessidade de cautela ao inferir que autoridade monetária brasileira, ao incluir o câmbio em sua função de reação, estabelece uma meta de estabilidade cambial.

Como agenda futura de pesquisa, buscar-se-á analisar a influência da coordenação de políticas monetárias e fiscais sobre a dinâmica da economia brasileira. Dessa forma, é possível avaliar a mudança das respostas dos juros frente a atuação mais expoente do lado fiscal do modelo. Também, podem-se incluir questões relativas a indexação de salários, bem como formação de hábito no consumo de segunda ordem, com o intuito de avaliar mais precisamente as questões de rigidezes nominais e reais no Brasil, e permitir uma melhor comparação com os estudos internacionais. 


\title{
Taylor rule and monetary policy in Brazil: empirical considerations from a DSGE model for a small-open economy
}

\begin{abstract}
The main objective of this paper is to analyze the Brazilian monetary policy after the inflation targeting adoption. Therefore, a Taylor rule is estimated from a DSGE model for a small open economy. The obtained results are compared with those presented on national literature, seeking for similarities and/or divergences. The conclusions of this essay are the following: (i) the presence of nominal and real rigidities is relevant to Brazilian economic dynamics, especially price indexation; (ii) the Central Bank of Brazil has an effective antiinflationary position; and (iii) the exchange rate is an auxiliary factor to the national monetary policy conduction.
\end{abstract}

Keywords: Monetary policy. Taylor rule. Bayesian estimation. DSGE.

\section{Regla de Taylor y la política monetaria en Brasil: consideraciones empíricas de un modelo DSGE para una economía pequeña y abierta}

\section{Resumen}

El objetivo principal de este trabajo es analizar la política monetaria de Brasil después de la adopción de metas de inflación. Por lo tanto, una regla de Taylor se estima a partir de un modelo DSGE para una economía pequeña y abierta. Los resultados obtenidos se compararon con los presentados en la literatura nacional, en busca de similitudes y/o divergencias. Las conclusiones de este trabajo son los siguientes: (i) la presencia de rigideces nominales y reales es relevante para la dinámica económica de Brasil, especialmente la indexación de precios; (ii) el Banco Central de Brasil tiene una posición anti-inflacionaria efectiva; y (iii) el tipo de cambio es un factor auxiliar a la conducción de la política monetaria nacional.

Palabras clave: Política monetaria. Regla de Taylor. Estimación bayesiana. DSGE. 


\section{Notas}

1 O termo DSGE foi originalmente utilizado por Kydland e Prescott (1982) em suas contribuições seminais à teoria dos Ciclos Reais (Real Business Cycles). Apesar de sua microfundamentação, os modelos RBC distinguem-se dos modelos DSGE ao incorporarem flexibilidade de preços, além de afirmarem que as flutuações macroeconômicas seriam provenientes apenas de choques reais. Desta forma, os mecanismos de transmissão convencionais da política monetária eram cerceados e a moeda considerada neutra.

2 Segundo Lucas (1976), os modelos macroeconômicos agregados até então utilizados seriam incapazes de demonstrar as implicações de políticas econômicas sobre os agentes. Isto porque tais políticas seriam capazes de modificar as expectativas destes agentes, alterando, portanto, os parâmetros dos modelos. Logo, as conclusões obtidas a partir dos modelos de sistemas de equações ad hoc não seriam verossímeis.

3 Estudos como os de Smets e Wouters (2003) e Adolfson et al. (2007) demonstraram que a capacidade preditiva dos modelos DSGE é significamente verossímel, senão melhor, em comparação à abordagem tradicional baseada em modelos de vetores autorregressivos bayesianos (BVAR).

4 Diversos modelos DSGE foram desenvolvidos pelos bancos centrais, como os modelos MAS (Chile), BEQM (Inglaterra), ToTEM (Canadá), NAWM (União Europeia) e SIGMA (EUA). Para mais detalhes, ver Harrison et al. (2005), Erceg, Guerrieri e Gust (2006), Medina e Soto (2006), Murchison e Rennison (2006) e Christoffel, Coenen e Warne (2007).

5 De modo geral, o conceito de pass-through cambial imperfeito estaria atrelado à incapacidade de os importadores reajustarem imediatamente os preços domésticos das importações frente a variações na taxa de câmbio. Para mais detalhes, ver Goldfajn e Werlang (2000).

6 Nesta versão, o modelo a ser estimado, formado por um sistema de equações não lineares, é linearizado em torno do seu nível de steady-state, com o intuito de se obter mais facilmente uma solução via métodos computacionais.

7 O compartilhamento imperfeito do risco está atrelado a restrições no crédito ou à falta de acesso a mercados de capitais, em que poderia ocorrer a comercialização de ativos e a mudança nos portfolios dos agentes.

8 No presente estudo, o modelo atém-se apenas à autoridade monetária para compor o governo, visto que a autoridade fiscal é tida apenas como um orçamento equilibrado, de forma que os impostos coletados sejam iguais ao subsídio requerido para eliminar a distorção induzida pela competição imperfeita nos mercados de bens domésticos e importados. Portanto, a estabilidade de preços é vista como objetivo principal e de responsabilidade exclusiva da autoridade monetária.

9 Para mais informações, consulte Lubik e Schorfheide (2005).

10 Para outras informações acerca dos métodos de estimação e solução de modelos DSGE, tidos como sistemas de equações em diferenças expectacionais não lineares, consultar Adjemian (2007).

11 O processo de filtragem é responsável por decompor os dados em um componente cíclico e um de tendência, sendo o primeiro implementado na estimação do modelo. Dessa forma, foca-se na frequência dos ciclos econômicos, visto que os modelos DSGE ajustam-se melhor ao paradigma de curto-prazo. Entretanto, DeJong e Dave (2007) afirmam que esse processo pode cercear a capacidade de previsão do modelo. Como o presente trabalho tem o objetivo de analisar a política monetária brasileira a partir das estimações dos parâmetros estruturais do modelo, não sua capacidade preditiva em si, o processo pôde ser aplicado sem efetiva perda de relevância.

12 Mais detalhes podem ser obtidos em Kim e Nelson (1999), Paulino, Turkman e Murteira (2003), Lancaster (2004) e Koop, Poirier e Tobias (2007).

13 Para obtenção das distribuições a posteriori dos parâmetros, utilizou-se o software Matlab ${ }^{\circledR} 7.12$ atrelado à plataforma Dynare 4.2 (ADJEMIAN et al., 2011).

\section{Referências}

ADJEMIAN, S. Bayesian estimation of DSGE models. 2007. Disponível em: <http://migre.me/ dEt0a>. Acesso em: out. 2014.

ADJEMIAN, S. et al. Dynare: Reference Manual, Version 4. Dynare Working Papers, 1, CEPREMAP, 2011. Disponível em: <http://www.dynare.org/wp-repo/dynarewp001.pdf>. Acesso em: jul. 2015.

ARAÚJO, L. A. D.; ANDRADE, J. P. Modelo DSGE e a Curva de Juros no Brasil. In: ENCONTRO REGIONAL DE ECONOMIA, 17, 2012, Fortaleza. Anais eletrônicos... Fortaleza: Teoria Econômica e Métodos Quantitativos. 2012. Disponível em: <http://edi.bnb.gov.br/content/aplicacao/eventos/ forumbnb2012/docs/sim_3_mesa4_modelo_dsge_curva_juros_brasil.pdf $>$. Acesso em: jul. 2015. 
CALVO, G. Staggered Prices in a Utility Maximizing Framework. Journal of Monetary Economics, v. 12, p. 383-398, 1983. Disponível em: <http://isites.harvard.edu/fs/docs/icb.topic500592. files/calvo.pdf>. Acesso em: jul. 2015.

CANOVA, F. Methods for Applied Macroeconomic Research. Princeton, NJ: Princeton University Press, 2007.

CASTRO, M. R. et al. SAMBA: Stochastic Analytical Model with a Bayesian Approach. Working Paper Series, Brasília, n. 239, p. 1-139, Apr. 2011.

CHRISTIANO, L. J.; EICHENBAUM, M.; EVANS, C. L. Nominal Rigidities and the Dynamic Effects of a Shock to Monetary Policy. Journal of Political Economy, Chicago, v. 113, n. 1, p. 1-45, 2005.

CHRISTOFFEL, K.; COENEN, G.; WARNE, A. Conditional versus Unconditional Forecasting with the New-Area-Wide Model of the Euro Area. Central Bank Workshop on Macroeconomic Modelling, Norges Bank, 2007.

CLARIDA, R.; GALÍ, J.; GERTLER, M. The Science of Monetary Policy: A New Keynesian Perspective. Journal of Economic Literature, Pittsburgh, v. 37, p. 1.661-1.707, 1999.

DEJONG, D. N.; DAVE, C. Structural Macroeconometrics. Princeton, NJ: Princeton University Press, 2007.

ERCEG, C.; GUERRIERI, L.; GUST, C. SIGMA: a New Open Economy Model for Policy Analysis. International Journal of Central Banking, San Francisco, v. 2, n. 1, p. 111-144, 2006.

FERREIRA, A. B. Metas para inflação e vulnerabilidade externa: um estudo do Brasil. 2004. 150f. Dissertação (Mestrado em Economia) - Centro de Desenvolvimento e Planejamento Regional, Universidade Federal de Minas Gerais, Belo Horizonte, 2004.

FURLANI, L. G. C.; PORTUGAL, M. S.; LAURINI, M. P. Constrained smoothing exchange rate moviments and monetary policy in Brazil: econometric and simulation evidence. Emerging Markets Review, v. 9, p. 247-265, 2008.

GALÍ, J.; MONACELLI, T. Monetary Policy and Exchange Rate Volatility in a Small Open Economy. The Review of Economic Studies Limited, Stockholm, v. 72, p. 707-734, 2005.

GOLDFAJN, I.; WERLANG, S. R.C. The pass-through from depreciation to inflation: a panel study. Working Paper Series, Brasília, n. 5, 2000.

GOODFRIEND, M. Interest Rates and the Conduct of Monetary Policy. Carnegie-Rochester Conference Series of Public Policy, Rochester, n. 34, p. 7-37, 1991.

HARRISON, R. et al. Bank of England Quarterly Model. Bank of England, 2005.

JUSTINIANO, A.; PRESTON, B. Monetary policy and uncertainty in an empirical small open economy model. Journal of Applied Econometrics, Chichester, v. 25, n. 1, p. 93-128, 2010.

KANCZUK, F. A Termometer for Macroprudential Policies. Working Paper Series, n. 4, 2012. Disponível em: <http://www.fea.usp.br/feaecon/RePEc/documentos/Kanczuk04WP.pdf>. Acesso em: jul. 2015.

KIM, C.; NELSON, C. R. State-Space Models with Regime Switching: Classical and Gibbs-Sampling Approaches with Applications. Massachussets: The MIT Press, 1999.

KOOP, G.; POIRIER, D. J.; TOBIAS, J. L. Bayesian Econometric Methods. Cambridge: Cambridge University Press, 2007.

KYDLAND, F.; PRESCOTT, E. Time to build and aggregate fluctuations. Econometrica, New York, v. 50, p. 1.345-1.370, 1982. 
LANCASTER, T. An Introduction to Modern Bayesian Econometrics. Oxford: Blackwell Publishing, 2004.

LUBIK, T.; SCHORFHEIDE, F. A Bayesian look at new open economy macroeconomics. In: GERTLER, M.; ROGOFF, K. (Ed.). NBER Macroeconomics Annual, 2005. p. 313-336.

LUCAS, R. E. Econometric Policy Evaluation: a critique. Carnegie-Rochester Conference Series on Public Policy, Rochester, v. 1, p. 19-46, 1976.

MEDINA, J. P.; SOTO, C. Model for analysis and simulations: a small open economy DSGE for Chile. Conference Paper, Central Bank of Chile, 2006.

MONACELLI, T. Monetary policy in a low pass-through environment. Journal of Money, Credit and Banking, Columbus, n. 37, p. 1.047-1.066, 2005.

MURCHISON, S.; RENNISON, A. ToTEM: The Bank of Canada's new quarterly projection model. Bank of Canada Technical Report, Ottawa, n. 97, p. 1-132, 2006. Disponível em: <http:// www.bankofcanada.ca/wp-content/uploads/2010/01/tr97.pdf>. Acesso em: jul. 2015.

PALMA, A. A.; PORTUGAL, M. S. Preferências do Banco Central do Brasil sob o regime de metas de inflação: estimação a partir de um modelo DSGE para uma pequena economia aberta. In: ENCONTRO BRASILEIRO DE FINANÇAS, 12, 2012, São Paulo. Anais... Rio de Janeiro: Sociedade Brasileira de Finanças, 2012.

PAULINO, C. D.; TURKMAN, M. A. A.; MURTEIRA, B. Estatística Bayesiana. Lisboa: Fundação Calouste Gulbenkian, 2003.

SILVA, F. S.; PORTUGAL, M. S. O impacto de choques fiscais na economia brasileira: uma abordagem DSGE. In: ENCONTRO BRASILEIRO DE ECONOMETRIA, 32, 2010, Salvador. Anais... Rio de Janeiro: Sociedade Brasileira de Econometria, 2010.

SILVEIRA, M. A. C. Two-country New Keynesian DSGE Model: a small open economy as a limit case. IPEA Discussion Paper, n. 1157, p. 1-48, 2006. Disponível em: <http://www.ipea.gov.br/ portal/images/stories/PDFs/TDs/td_1157.pdf>. Acesso em: jul. 2015.

. Using a Bayesian Approach to Estimate and Compare New Keynesian DSGE Model for the Brazilian Economy: the Role for Endogenous Persistence. Revista Brasileira de Economia, Rio de Janeiro, v. 62, n. 3, p. 333-357, 2008. Disponível em: <http://www.scielo.br/pdf/rbe/v62n3/ a05v62n3.pdf>. Acesso em: jul. 2015.

SIN, H. L.; GAGLIANONE, W. P. Stochastic simulation of a DSGE model for Brazil. MPRA Paper, Munich, n. 20.853, p. 1-40, 2006.

SMETS, F.; WOUTERS, R. An Estimated Stochastic Dynamic General Equilibrium Model of the Euro Area. Journal of the European Economic Association, Hoboken, v. 1, p. 1123-1175, 2003.

Comparing shocks and frictions in US and Euro business cycles: a bayesian DSGE approach. Journal of Applied Econometrics, Chichester, v. 20, n. 2, p. 161-183, 2005.

VALLI, M.; CARVALHO, F. A. Fiscal and monetary policy interaction: a simulation based analysis of a two-country new keynesian DSGE model with heterogeneous households. Working Paper Series, Brasília, n. 204, p. 1-114, Apr. 2010.

WALQUE, G.; SMETS, F.; WOUTERS, R. An Open Economy DSGE Model Linking the Euro Area and the US Economy. National Bank of Belgium, Bruxelas, 2005. Manuscrito. 


\section{APÊNDICE}

Tabela A - Distribuição a posteriori dos parâmetros do modelo completo

\begin{tabular}{|c|c|c|c|}
\hline Parâmetro & Média a priori & Média a posteriori & Intervalo de confiança \\
\hline \multicolumn{4}{|c|}{ Preferências domésticas } \\
\hline $\mathrm{h}$ & 0,70 & 0,7571 & {$[0,6721 ; 0,8359]$} \\
\hline$\sigma$ & 1,00 & 1,2646 & {$[1,1239 ; 1,3779]$} \\
\hline$\varphi$ & 2,00 & 1,7959 & {$[1,5782 ; 1,9311]$} \\
\hline$\eta$ & 0,60 & 0,5221 & {$[0,4682 ; 0,6020]$} \\
\hline \multicolumn{4}{|c|}{ Determinação de preços domésticos } \\
\hline$\delta_{H}$ & 0,70 & 0,3615 & {$[0,1652 ; 0,5170]$} \\
\hline$\delta_{F}$ & 0,70 & 0,7967 & {$[0,6537 ; 0,9316]$} \\
\hline$\theta_{H}$ & 0,50 & 0,9904 & {$[0,9864 ; 0,9952]$} \\
\hline$\theta_{F}$ & 0,50 & 0,8720 & {$[0,8221 ; 0,9155]$} \\
\hline \multicolumn{4}{|c|}{ Política monetária doméstica } \\
\hline$\rho_{i}$ & 0,50 & 0,4363 & {$[0,3193 ; 0,5722]$} \\
\hline$\psi_{\pi}$ & 1,50 & 1,2540 & {$[1,0405 ; 1,5997]$} \\
\hline$\psi_{y}$ & 0,25 & 0,6441 & {$[0,5894 ; 0,7289]$} \\
\hline$\psi_{q}$ & 0,25 & 0,5455 & {$[0,4413 ; 0,6190]$} \\
\hline \multicolumn{4}{|c|}{ Preferências externas } \\
\hline$h^{*}$ & 0,70 & 0,9947 & {$[0,9935 ; 0,9960]$} \\
\hline$\sigma^{*}$ & 1,00 & 0,9989 & {$[0,8435 ; 1,1995]$} \\
\hline$\varphi^{*}$ & 2,00 & 1,5378 & {$[1,2049 ; 1,8120]$} \\
\hline \multicolumn{4}{|c|}{ Política monetária externa } \\
\hline$\rho_{i}^{*}$ & 0,50 & 0,1324 & {$[0,0396 ; 0,2262]$} \\
\hline$\psi_{\pi}^{*}$ & 1,50 & 2,4081 & {$[2,2285 ; 2,5761]$} \\
\hline$\psi_{y}^{*}$ & 0,25 & 0,1481 & {$[0,0754 ; 0,2226]$} \\
\hline
\end{tabular}




\begin{tabular}{|c|c|c|c|}
\hline \multicolumn{4}{|c|}{ Persistência dos choques domésticos e externos } \\
\hline$\rho_{a}$ & 0,50 & 0,8240 & {$[0,7280 ; 0,9050]$} \\
\hline$\rho_{G}$ & 0,50 & 0,6536 & {$[0,4280 ; 0,8276]$} \\
\hline$\rho_{q}$ & 0,50 & 0,8283 & {$[0,7087 ; 0,9050]$} \\
\hline$\rho_{\alpha}^{*}$ & 0,50 & 0,9918 & {$[0,9860 ; 0,9983]$} \\
\hline$\rho_{G}^{*}$ & 0,50 & 0,9990 & {$[0,9985 ; 0,9994]$} \\
\hline \multicolumn{4}{|c|}{ Desvios-padrão dos choques domésticos e externos } \\
\hline$\sigma_{a}$ & 3,00 & 4,1135 & {$[1,3806 ; 6,4465]$} \\
\hline$\sigma_{q}$ & 1,00 & 0,1672 & {$[0,1347 ; 0,2009]$} \\
\hline$\sigma_{G}$ & 1,00 & 0,2236 & {$[0,1787 ; 0,2639]$} \\
\hline$\sigma_{a}^{*}$ & 3,00 & 0,6656 & {$[0,4747 ; 0,8133]$} \\
\hline$\sigma_{G}^{*}$ & 1,00 & 0,3279 & {$[0,2384 ; 0,4478]$} \\
\hline$\sigma_{M}$ & 1,00 & 0,1472 & {$[0,1256 ; 0,1701]$} \\
\hline$\sigma_{M}^{*}$ & 1,00 & 0,5787 & {$[0,4570 ; 0,6931]$} \\
\hline
\end{tabular}

Fonte: elaboração do autor. 